\title{
Cosmic rays and the magnetic field of the nearby starburst galaxy NGC 253
}

\author{
Volker Heesen and Ralf-Jürgen Dettmar \\ Ruhr-University Bochum, Germany \\ E-mail: heesendastro.rub.de, dettmareastro.rub.de
}

\section{Marita Krause and Rainer Beck}

Max Planck Institute for Radio Astronomy, Germany

E-mail: mkrause@mpifr-bonn.mpg.de, rbeck@mpifr-bonn.mpg.de

Using radio polarimetry we study the connection between the transport of cosmic rays (CR's), the three-dimensional magnetic field structure, and features of other ISM phases in the halo of NGC 253. We present a new sensitive radio continuum map of NGC 253 obtained from combined VLA and Effelsberg observations at $\lambda 6.2 \mathrm{~cm}$. We find a prominent radio halo with a scaleheight of the thick radio disk of $1.7 \mathrm{kpc}$. The linear dependence between the local scaleheight of the vertical continuum emission and the cosmic ray electron (CRE) lifetime requires a vertical $\mathrm{CR}$ bulk speed of $270 \mathrm{~km} \mathrm{~s}^{-1}$.

The magnetic field structure of NGC 253 resembles an " $X$ "-shaped configuration where the orientation of the large-scale magnetic field is plane-parallel only in the inner regions of the disk and at small distances from the galactic midplane. At larger galactocentric radii and further away from the midplane the vertical component becomes important. This is most clearly visible at the location of the "radio spur" southeast of the nucleus, where the magnetic field orientation is almost vertical. We made a simple model for the dominant toroidal $(r, \phi)$ magnetic field component using a spiral magnetic field with prescribed inclination and pitch angle. The residual poloidal $(r, \phi, z)$ magnetic field component which was revealed by subtracting the model from the observations shows a distinct " $X$ "-shaped magnetic field orientation centered on the nucleus. The orientation angle of the poloidal magnetic field is consistent with a magnetic field transport described by the superposition of the vertical CR bulk speed and the rotation velocity.

Hence, we propose a disk wind which transports cosmic rays, magnetic field, and (partially) ionized gas from the disk into the halo.

From planets to dark energy: the modern radio universe

October 1-5 2007

University of Manchester, Manchester, UK 


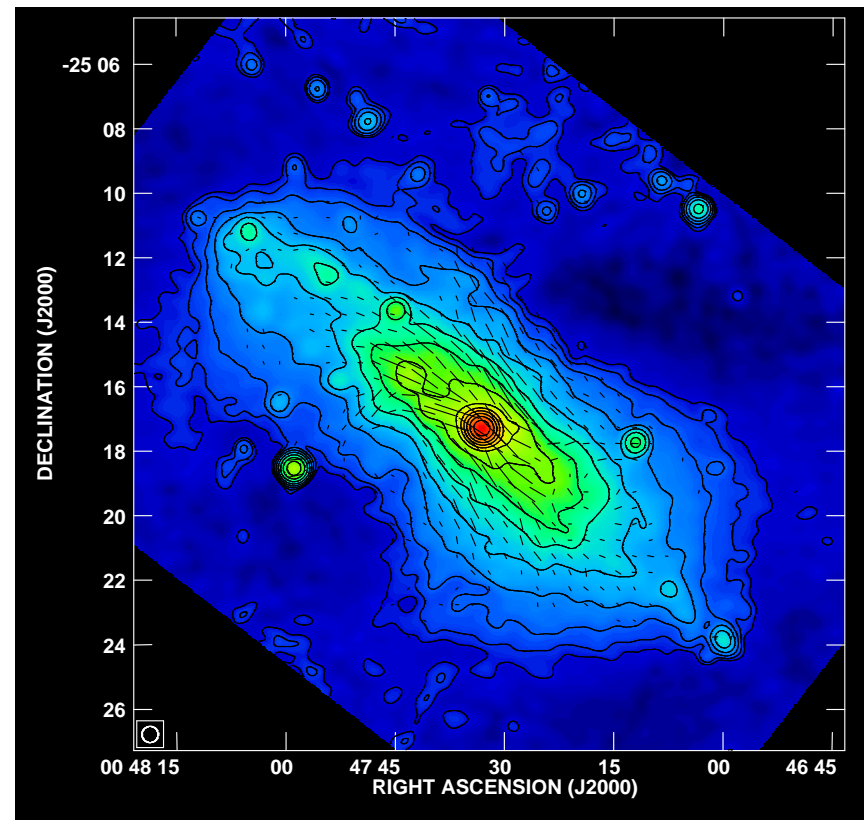

Figure 1: Total power radio continuum obtained from the combined VLA + Effelsberg observations at $\lambda 6.2 \mathrm{~cm}$. The overlaid vectors indicate the intrinsic orientation of the resolved regular magnetic field. The length of the vectors is defined by 1 arcsec to be equivalent to $12.5 \mu \mathrm{Jy} /$ beam of polarized intensity. Contours are at 3, 6, 12, 24, 48, 96, 192, 384, 768, 1536, 3077, 6144, 12288 , and $24576 \times 30 \mu \mathrm{Jy} /$ beam. The size of the beam is $30 \operatorname{arcsec}$ (HPBW).

\section{Observations}

We have conducted an extensive radio continuum multiwavelength study both with the VLA interferometer ${ }^{1}$ and the $100-\mathrm{m}$ Effelsberg telescope ${ }^{2}$. A mosaic at $\lambda 6.2 \mathrm{~cm}$ consisting of 15 pointings has been observed with the VLA. The strong nuclear continuum point source has been taken into account in our data reduction: we cleaned our single-dish maps in order to correct for sidelobes and applied a correction for the instrumental polarization. The instrumental polarization caused by the off-axis placement of the nuclear point source in the VLA primary beam has been addressed by a specially tailored polarization calibration. The missing zero-spacing flux of the VLA mosaic has been filled up with the $\lambda 6.2 \mathrm{~cm}$ Effelsberg map.

\section{The cosmic ray distribution}

We find a prominent radio halo with a thin and a thick radio disk with a scaleheight of $0.3 \mathrm{kpc}$ and $1.7 \mathrm{kpc}$ at $\lambda 6.2 \mathrm{~cm}$, respectively. The steepening of the spectral index with increasing distance from the disk suggests synchrotron radiation to be the dominant energy loss of the cosmic ray electrons (CRE's). The linear dependence between the local scaleheight and the CRE lifetime requires a vertical CR bulk speed of $(270 \pm 30) \mathrm{km} \mathrm{s}^{-1}$. This explains the dumbbell shape visible in the total power distribution presented in Fig. 1, where the smallest scaleheights are found in the inner regions of the disk where the magnetic field strength is largest. The CR bulk speed is close to the escape velocity of $v_{\mathrm{esc}}=280 \mathrm{~km} \mathrm{~s}^{-1}$, where we used $v_{\mathrm{esc}}=\sqrt{2} \cdot v_{\text {rot }}$ with a rotation velocity of $v_{\text {rot }}=200 \mathrm{~km} \mathrm{~s}^{-1}$. The kinetic energy of the CR gas is thus possibly high enough to drive a galactic wind which eventually leaves the gravitational potential.

\footnotetext{
${ }^{1}$ The VLA (Very Large Array) is operated by the NRAO (National Radio Astronomy Observatory).

${ }^{2}$ The Effelsberg 100-m telescope is operated by the Max-Planck Institut für Radioastronomie (MPIfR).
} 


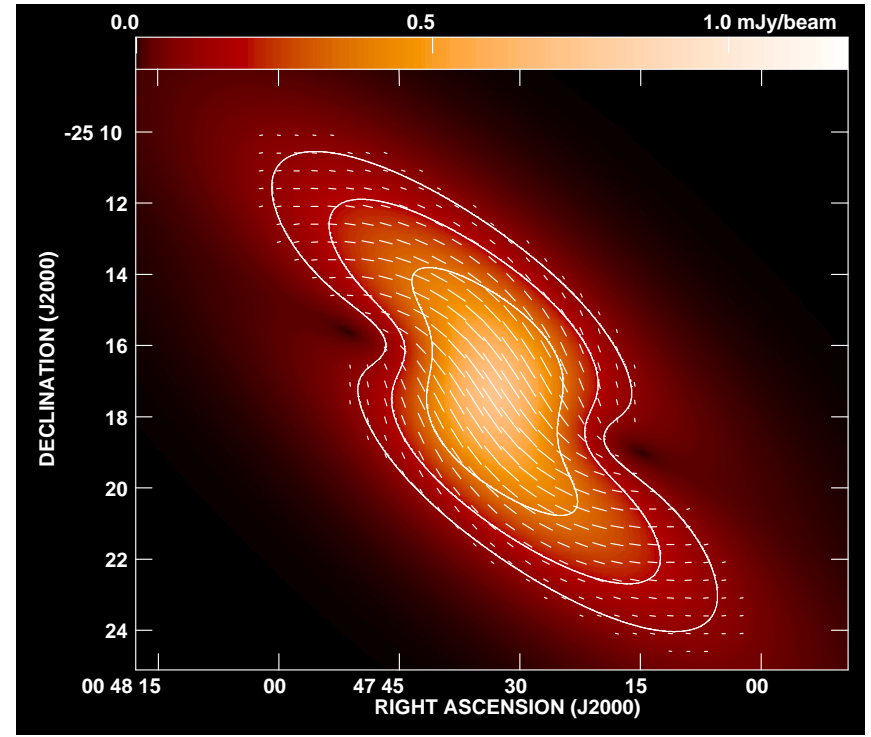

Figure 2: Axisymmetric model for the toroidal magnetic field with a pitch angle of $25^{\circ}$ of the magnetic field spiral. Contours are at 3,6 , and $12 \times 30 \mu \mathrm{Jy} /$ beam. The overlaid vectors indicate the intrinsic orientation of the resolved regular magnetic field. The length of the vectors is defined by 1 arcsec to be equivalent to $12.5 \mu \mathrm{Jy} /$ beam polarized intensity. The size of the beam is $30 \operatorname{arcsec}$ (HPBW).

\section{The magnetic field structure}

Using the revised equipartition formula of [1] we derive a total magnetic field strength in the disk between $9 \mu \mathrm{G}$ (outer part) and $16 \mu \mathrm{G}$ (inner part). The distribution of the Rotation Measure (RM) shows the disk and halo to be split in a positive northeastern and negative southwestern part. From the comparison with the velocity field following the method described in [2] we find the radial component of the spiral magnetic field to be directed inwards. Hence, we constructed a simple model for the large-scale magnetic field using an axisymmetric spiral magnetic field with a prescribed inclination and pitch angle (Fig. 2). We find good agreement between the model and the observed magnetic field configuration. In order to get the distribution of the poloidal magnetic field we subtracted the model from the observed polarization. We find an "X"-shaped pattern centered on the nucleus which correlates well with the extra-planar $\mathrm{H} \alpha$ emission and soft X-ray emission. This significant poloidal magnetic field component supports the transport of cosmic rays along the field lines connecting the disk with the halo. The orientation angle of the poloidal magnetic field can be explained by a disk wind blowing from the disk into the halo as the superposition of the vertical CR bulk speed and the rotation velocity of the disk. This work will be fully presented in [3], [4], and [5].

\section{References}

[1] R. Beck \& M. Krause, Revised equipartition and minimum energy formula for magnetic field strength estimates from radio synchrotron observations, Astr. Nachr., 326 (2005) 414-427

[2] F. Krause \& R. Beck, Symmetry and direction of seed magnetic fields in galaxies, A\&A, 335 (1998) 789-796

[3] V. Heesen, Cosmic rays and the magnetic field in the nearby starburst galaxy NGC 253, PhD thesis, Ruhr-University Bochum, Germany (2008)

[4] V. Heesen, R. Beck, M. Krause, \& R.-J. Dettmar, Cosmic rays and the magnetic field in the nearby starburst galaxy NGC 253 I. The cosmic ray distribution, $A \& A$ (2008), in prep.

[5] V. Heesen, M. Krause, R. Beck, \& R.-J. Dettmar, Cosmic rays and the magnetic field in the nearby starburst galaxy NGC 253 II. The magnetic field structure, A\&A (2008), in prep. 